\title{
Drainage Procedures for the Treatment of Walled-Off Pancreatic Necrosis: Is More Refinement Necessary?
}

\author{
Sung-Hoon Moon \\ Department of Internal Medicine, Hallym University Sacred Heart Hospital, Hallym University College of Medicine, Anyang; Korea
}

See "Outcomes of Infected versus Symptomatic Sterile Walled-Off Pancreatic Necrosis Treated with a Minimally Invasive Therapy" by Jong Jin Hyun, et al. on page 215, Vol. 13. No. 2, 2019

Pancreatic necrosis (PN) develops in 5\% to 40\% of patients with acute pancreatitis despite adequate treatment in the early phase. ${ }^{1-4}$ According to its location, PN can involve the pancreatic parenchyma, the peripancreatic parenchyma, or both. The most important factor in determining invasive treatment for PN is the presence of infection in the necrotic tissue. In cases with asymptomatic sterile PN, conservative management is the treatment of choice. However, when infection of PN is diagnosed by a positive aspirate, surgical necrosectomy has been the standard treatment for decades, based on the surgical dogma of removing the necrotic or dead tissue that is prone to infection. ${ }^{3}$ From the 1980s to the early 1990s, infected PN had been regarded as an indication for immediate (within 24 hours) open surgical necrosectomy. ${ }^{5}$ Since then, however, the concept of minimally invasive intervention and the notion of postponing intervention have been supported by a variety of studies.

The latest evidence-based guidelines recommend a "staged," "minimally invasive," "step-up" approach for patients with infected necrotizing pancreatitis (Table 1). ${ }^{2,3,5}$ In clinical practice, infected PN is diagnosed when gas is found in the necrotic collection in an imaging study and/or when unequivocal clinical signs of infection are present without another infectious focus (i.e., pneumonia). ${ }^{3,7}$ However, gas may also occur in the necrotic collection as a result of a fistula to the stomach/intestine. The use of fine needle-aspiration (FNA) for diagnostic purposes alone is limited in a state-of-the-art practice. ${ }^{2-4,7}$ When infected PN is suspected, the recommended treatment is administration of broad spectrum antibiotics with optimal penetration, as antibiotics may postpone or even obviate the need for invasive intervention. If antibiotics fail to ameliorate clinical deteriora- tion, the next consideration is catheter drainage of the necrotic collection, as drainage of the infected fluid may stabilize the patient's clinical condition and provide time for further encapsulation. Gram staining and culture of the fluid obtained during initial drainage may also guide the use of appropriate antibiotics. Many patients can be successfully treated with catheter drainage alone, without the need for additional necrosectomy; therefore, necrosectomy can be reserved for those cases in which infected PN cannot be managed with catheter drainage. Compared with open surgical necrosectomy, minimally invasive necrosectomy induces a smaller proinflammatory response and a lower rate of new-onset organ failure, thereby resulting in lower mortality and morbidity. ${ }^{3}$

Invasive intervention for infected PN should ideally be postponed until the necrotic collection has partially liquefied and has become encircled by a well-defined inflammatory wall. This walled-off necrosis (WON) usually occurs >4 weeks after the onset of necrotizing pancreatitis and can allow a safer and more effective intervention., ${ }^{4,7}$ The interventions used to drain and/or debride pancreatic/peripancreatic necrosis can be categorized into: (1) endoscopic approaches-endoscopic drainage with/ without subsequent endoscopic necrosectomy; (2) percutaneous approaches-percutaneous catheter drainage with/without subsequent minimally invasive retroperitoneal surgery (i.e., videoscopic-assisted retroperitoneal debridement or sinus tract endoscopy). Open surgical approaches are seldom used any more. The treatment modality is chosen by multidisciplinary consensus based on the center's expertise, facilities, and clinical experience, as well as the location of the PN.

In this issue of Gut and Liver, the Virginia Mason Medical

Correspondence to: Sung-Hoon Moon (https://orcid.org/0000-0002-7879-3114)

Department of Internal Medicine, Hallym University Sacred Heart Hospital, Hallym University College of Medicine, 22 Gwanpyeong-ro 170beon-gil, Dongan-gu, Anyang 14068, Korea

Tel: +82-31-380-3710, Fax: +82-31-386-2269, E-mail: endomoon@naver.com

pISSN 1976-2283 eISSN 2005-1212 https://doi.org/10.5009/gnl19055

(a) This is an Open Access article distributed under the terms of the Creative Commons Attribution Non-Commercial License (http://creativecommons.org/licenses/by-nc/4.0) which permits unrestricted non-commercial use, distribution, and reproduction in any medium, provided the original work is properly cited. 
Table 1. Management of Necrotic Collection in Acute Necrotizing Pancreatitis

\begin{tabular}{lll}
\hline & Acute necrotic collection (during the first 4 weeks) & Walled-off necrosis (after 4 weeks) \\
$\begin{array}{l}\text { Indication of invasive } \\
\text { intervention for necrotic }\end{array}$ & Infection & Infection \\
collection & & Obstruction of stomach/intestine \\
Staged step-up approach & Step 1. Conservative medical treatment (i.e., antibiotics) & Step 1. Conservative medical treatment (i.e., antibiotics) \\
& Step 2. Percutaneous catheter drainage* & Step 2. Endoscopic or percutaneous drainage \\
& Step 3. Additional drainage or upsizing of the catheter & Step 3. Additional drainage or upsizing of the catheter \\
& & Step 4. Endoscopic necrosectomy or minimally invasive \\
& & retroperitoneal necrosectomy \\
& & Step 5. Repeat procedure if necessary
\end{tabular}

Concept of the minimally invasive step-up approach

- The step-up approach consists of an initial minimally invasive treatment, followed by a gradually more invasive procedure if the initial treatment fails.

- The aim is to delay intervention whenever possible.

- A minimally invasive treatment induces less intervention-related stress, whereas surgical necrosectomy induces a proinflammatory immune response and new-onset organ failure in patients who are already critically ill.

*Placement of a catheter before encapsulation may be debatable, but encapsulation is not "sine qua non" for catheter drainage.

Center group report on the outcomes of dual modality drainage in patients with symptomatic WON. ${ }^{8}$ The authors also confirmed that patient outcomes were worse for infected WON than for symptomatic sterile WON. The dual modality drainage consisted of percutaneous drainage, followed immediately by placement of endoscopic transmural stent(s). This dual modality drainage may not be fully consistent with a typical step-up approach, because the second drainage in a step-up approach is generally performed only if no clinical improvement is seen 72 hours after first drainage. ${ }^{5}$ Therefore, a strict requirement for dual modality drainage in all their patients with symptomatic WON is questionable. Moreover, endoscopic drainage may only be feasible in patients whose WON is located adjacent to the gastric or duodenal wall, and who are also in relatively good general condition and can tolerate a complex endoscopic procedure.

Interestingly, the cases of symptomatic sterile WON ( $\mathrm{n}=113)$ outnumbered the infected WON ( $n=93)$ in the Virginia Mason group's study population who underwent the invasive intervention. In cases with sterile WON, the invasive intervention can be performed only for unrelenting pain or obstruction of the gastric outlet, intestine, or bile duct, because catheter drainage of sterile WON has a potentially grave risk of introducing infection. ${ }^{3,5}$ The followings are plausible explanations for the relatively large number of sterile WON in this study population. First, selection biases may result from the eligibility for dual modality drainage. Second, referral biases may influence the study population. Third, the authors used a strict definition of infected WON based on the positive culture from fluid aspirates obtained during initial placement of drainage. However, the reported false negative rate of FNA in this setting can be up to $25 \%$. $^{2,3,5,7}$
The authors reported a remarkably low mortality (2.4\%/4.1\%) in patients with symptomatic/infected WON who underwent the invasive intervention. The study population did not include early-phase deaths from acute necrotizing pancreatitis. Regarding the timing of mortality in severe acute pancreatitis, approximately half of these deaths occur within 14 days after the onset of symptoms due to organ failure. ${ }^{9}$ Nevertheless, the favorable clinical outcomes of this study, from the aspects of low mortality and morbidity, are very notable, particularly as necrosectomy was not performed in any of their 211 patients with symptomatic WON. The reported rates of necrosectomy performed after catheter drainage varies markedly, from 0 to $100 \%$ among centers. ${ }^{3}$ The timing of necrosectomy may vary among centers because the clinicians decide whether patients show any improvement in their clinical deterioration, and this may influence the necrosectomy rate.

Dual modality drainage, which is a modified form of drainage procedures, may have the potential to decrease the need for necrosectomy. In addition, dual modality drainage may have the advantage of avoiding the occurrence of pancreaticocutaneous fistula. Chronic pancreaticocutaneous fistula is a feared complication of percutaneous drainage in the setting of necrotizing pancreatitis, especially with a disconnected pancreatic duct. ${ }^{10}$ No chronic pancreaticocutaneous fistula developed in any patient who was treated with dual modality drainage, since this method provided an outlet for fluid passage into the gastrointestinal tract.

Minimally invasive step-up approaches have markedly decreased the late-phase deaths of acute necrotizing pancreatitis, whereas modern critical care has apparently reduced the early- 
phase deaths. Refined drainage procedures have further shifted the paradigm from necrosectomy to drainage. Refined drainage procedures include placement of multiple percutaneous catheters, catheter upsizing, multiple transluminal gateways, and dual modality techniques. Given the single-digit mortality rate already achieved for necrotizing pancreatitis, the drainage procedures may need more refinements to provide further decreases in factors such as the necrosectomy rate, number of procedures, procedure-related adverse events, length of hospitalization, duration of drainage, and hospital costs.

\section{CONFLICTS OF INTEREST}

No potential conflict of interest relevant to this article was reported.

\section{REFERENCES}

1. Crockett SD, Wani S, Gardner TB, Falck-Ytter Y, Barkun AN; American Gastroenterological Association Institute Clinical Guidelines Committee. American Gastroenterological Association Institute guideline on initial management of acute pancreatitis. Gastroenterology 2018;154:1096-1101.

2. Isayama $H$, Nakai $Y$, Rerknimitr R, et al. Asian consensus statements on endoscopic management of walled-off necrosis Part 1: epidemiology, diagnosis, and treatment. J Gastroenterol Hepatol
2016;31:1546-1554.

3. van Grinsven J, van Santvoort HC, Boermeester MA, et al. Timing of catheter drainage in infected necrotizing pancreatitis. Nat Rev Gastroenterol Hepatol 2016;13:306-312.

4. Banks PA, Bollen TL, Dervenis C, et al. Classification of acute pancreatitis--2012: revision of the Atlanta classification and definitions by international consensus. Gut 2013;62:102-111.

5. Bugiantella W, Rondelli F, Boni M, et al. Necrotizing pancreatitis: a review of the interventions. Int J Surg 2016;28 Suppl 1:S163S171.

6. da Costa DW, Boerma D, van Santvoort HC, et al. Staged multidisciplinary step-up management for necrotizing pancreatitis. Br J Surg 2014;101:e65-e79.

7. Freeman ML, Werner J, van Santvoort HC, et al. Interventions for necrotizing pancreatitis: summary of a multidisciplinary consensus conference. Pancreas 2012;41:1176-1194.

8. Hyun JJ, Sahar N, Singla A, et al. Outcomes of infected versus symptomatic sterile walled-off pancreatic necrosis treated with a minimally invasive therapy. Gut Liver 2019;13:215-222.

9. Fu CY, Yeh CN, Hsu JT, Jan YY, Hwang TL. Timing of mortality in severe acute pancreatitis: experience from 643 patients. World J Gastroenterol 2007;13:1966-1969.

10. Jang JW, Kim MH, Oh D, et al. Factors and outcomes associated with pancreatic duct disruption in patients with acute necrotizing pancreatitis. Pancreatology 2016;16:958-965. 